housing and sheltered accommodation options in the lockdown. The 70\% male preponderance of Q1-2019/20 is due to 7 male UASC. The Q1-2020/21 is more akin to annual national data. Our cohort shows a significant rise in Gypsy/ Roma and other White European ethnicity and an increase in belonging to a sibling group of 3 or more. We note significant rises in child illness/disability, parental substance misuse, domestic abuse, parental illness/disability and family in acute stress recorded as reasons for entering care. This may be due to a deterioration during lockdown, with sudden and unexpected change in provision of services, reduced resources, increased pressure on finance and family dynamics amplified by the pandemic. This significant rise of LAC in Q1-2020/21 impacts on the quality and financial aspects for Health/Social Care in the short, medium and long term.

\section{Quality improvement and patient safety}

\section{HOW EFFECTIVE HAVE 'CHILDREN'S CRITICAL CARE CASE REVIEW MEETINGS (C4RM)' BEEN IN IMPROVING PATIENT CARE?}

Dineth Wijesiriwardena, Shahida Ahmed, Maimuna Mushabe, Louise Tebble. Mid and South Essex University Hospitals NHS Trust

\subsection{6/archdischild-2021-rcpch.19}

Background C4RM involves critical review of management of all paediatric cases that require transfer to intensive care unit by completing a 'harm review proforma' for all such cases using root cause analysis methodology. All paediatric cases that require transfer to intensive care unit are 'Incident reported' so they can be identified. A weekly meeting is conducted to go through all the cases. One/two interesting cases are selected for presentation at the monthly C4RM meeting.

All teams involved in patient's care are invited to attend. Areas of good practice as well as those requiring improvement and any contributory factors leading to deviation from best practice are identified. Actions are agreed based on recommendations which are widely shared and displayed. C4RM report is also fed into monthly Governance meeting.

Objectives To improve patient care by learning from our experiences, sharing best practice, developing/improving policies and working together.

Methods After conducting C4RM meetings, auditing was performed to see if there were improvements with regards to the sepsis pathway and number of serious incidents. In addition, reviews were performed to identify areas that required improvement.

Results C4RM meetings have been instrumental in increasing awareness about factors that are involved in improving patient care.

- To improve patient care in Paediatric assessment unit (PAU), 'Short Stay policy' and 'Re-attender policy' were developed. Safety huddles with Emergency Department (ED) were introduced.

- Sepsis pathway was audited and amendments were made to make it more suitable for use in ED and PAU. A sustained improvement in Sepsis Audit results was seen (69\% compliance in Jan 18 to $89 \%$ in Dec18).

- Joint working was enhanced with teams including Orthopaedic, surgeons, anaesthetists, ED and pathways were streamlined.

- 'Difficult Airway pathway' and 'Difficult intubation trolley' were set up with input from Anaesthetic team and ENT. 'Time Critical transfer pathway' and 'STOPP transfer tool' were implemented with input from Anaesthetic team.

- Relationships have been strengthened with Children's retrieval service with 'Outreach study days' being organised locally.

- A sustained reduction in number of Serious Incidents was noticed over the years $(2016 / 17=15,2017 / 18=5,2018 / 19$ $=5)$.

- Documentation and communication improved by implementing 'SBAR tool', 'Tertiary communication forms' and strict adherence with 'CEWT scoring tool'.

Conclusions C4RM meetings have been an innovative and effective means of improving patient care. These meetings have received immense appreciation and a neighbouring Network has requested to help it roll out to the entire network.

\section{British Association for Community Child Health}

\section{ADHERENCE OF SRI LANKAN PRESCHOOL CHILDREN TO THE WHO 24-HOUR MOVEMENT GUIDELINES FOR EARLY YEARS AND ITS ASSOCIATIONS- SUNRISE PILOT STUDY}

${ }^{1}$ Panagoda Weththasinghage Prasad Chathurangana, ${ }^{2}$ Anthony D Okely, ${ }^{1}$ Vithanage Pujitha Wickramasinghe. ${ }^{1}$ Faculty of Medicine, University of Colombo; ${ }^{2}$ Early Start, Faculty of Arts, Social Sciences and Humanities, University of Wollongong, Australia

\subsection{6/archdischild-2021-rcpch.20}

Background Physical inactivity is a major contributor to overweight, obesity and death. Early childhood is a critical time to promote healthy lifestyle behaviours. World Health Organisation (WHO, 2019) recommends $1 . \geq 180$ minutes of total physical activity (TPA), including $\geq 60$ minutes of moderate-tovigorous physical activity (MVPA), 2. $\leq 1$ hour of restrained seating (RS) and $\leq 1$ hour of screen time (ST) 3. 10-13 hours of sleep for this age group.

Objectives The purpose of this study was to determine the proportion of Sri Lankan preschool children complying with individual and combined guidelines and various associated factors.

Methods Participants were recruited from 8 nurseries in the Colombo District, Western province. A self-administered questionnaire collected demographical data, sleep time and screen time. ActiGraph GT3X ${ }^{\oplus}$ accelerometers were used to collect data on physical activity over 72 hours. The gross and fine motor development were assessed by Ages \& Stages Questionnaires (3rd Edition) and executive functions were assessed by the Early Years Toolbox on iPad. Data were analysed by SPSS version 23. Results Ninety-nine children (mean age: 4.81 years, 45 boys and 54 girls) had complete data. The proportions of adhering 
to TPA, MVPA, RS, ST and Sleep time recommendations were $53 \%, 84 \%, 84 \%, 53 \%$ and $65 \%$ respectively. The mean MVPA and TPA durations were longer in boys $(p=0.001$ and $\mathrm{p}=0.046)$. Girls adhered to screen time more than boys $(p=0.007)$. Children from rural areas complied with sleep time more than urban areas $(\mathrm{p}=0.048)$. Only $18 \%$ complied with all the guidelines. There was no association between adhering to individual or combined recommendations and BMI, gross and fine motor scores or executive functions.

Conclusions Less than a fifth of pre-schoolers met all the recommendations. Future work should focus on reducing screen time and increasing physical activity.

\section{Association of Paediatric Emergency Medicine}

\section{IMPROVING EDUCATION IN THE CHILDREN'S EMERGENCY DEPARTMENT THROUGH THE IMPLEMENTATION OF A JOURNAL CLUB}

Laura Lee. NNUH

\subsection{6/archdischild-2021-rcpch.21}

Background Anecdotally from discussion with staff in the children's emergency department; it was suspected that staff were infrequently engaging in $\mathrm{CPD}$, in particular reading journal articles. Much of the CPD that the team were participating in was mandatory and with the emergence of the pandemic much of this training was stopped.

It was decided to set up a journal club in order to improve this.

Objectives To improve the rate in which staff are reading journal articles through the implementation of a journal club in a children's emergency department.

Methods This was a service improvement audit project which used some short surveys to ascertain staff exposure to journals prior to the implementation of the journal club and 3 months following its implementation.

The survey was distributed to the whole team via social media and email and the whole team were encouraged to complete, with $54 \%$ of the team completing each time.

Journal club was implemented via teams once a month for an hour where 2 papers were presented and discussed. The plan was to initially keep it basic to encourage multidisciplinary engagement but then to build up the amount of critical appraisal over time; 'tell us what your article is, what your article is about and why you like it.'

Results Attendance at journal club has ranged form 4 per session to 10 per session and is dependant on shifts to enable participation. The participants have been from medical and nursing background and a variety of grades and roles; including health care assistants, nurses and medical team members.

The initial audit showed that $22 \%$ of staff were never reading journal articles and a further $17 \%$ only read once per year.

$44 \%$ were reading at least monthly.

The reasons for not reading articles included the following; not knowing where to search, not having time, finding credible articles, the length of the article, lots of statistics.
The results from the second survey showed that 18\% of staff were never reading articles and $12 \%$ reading yearly.

$64 \%$ of staff are reading at least monthly since the implementation of journal club.

$100 \%$ of respondents were keen for journal club to continue. 1 respondent wanted some teaching in the use of technology and in order to enable better access the team wanted the whole year dates published in order to request shifts in enable attendance. Articles have also not only been shared electronically but have been made available in hard copy to enable access to all.

Conclusions The implementation of a journal club has been well received and participated in with $100 \%$ of respondents suggesting it continues. $20 \%$ more staff have read articles at least monthly since its implementation. However there has only been a $3 \%$ improvement in those that are never reading articles. It is necessary to further address this starting with the production of an infographic further reinforcing what journal club is about and how it works and some further auditing later down the line.

\section{Association of Paediatric Emergency Medicine}

\section{2 RECOGNITION OF LEG PAIN AS A RED FLAG SYMPTOM FOR MENINGOCOCCAL SEPTICAEMIA IN CHILDREN}

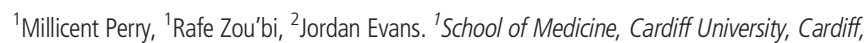
UK; ${ }^{2}$ Paediatric Emergency Department, University Hospital of Wales, Cardiff, UK

\subsection{6/archdischild-2021-rcpch.22}

Background The mortality and morbidity of meningococcal septicaemia, a leading cause of septic shock in children, can be reduced by early recognition and diagnosis. NICE guidance [CG102] identifies leg/limb pain as a high-risk symptom of meningococcal septicaemia. Recently, the Petechiae in Children (PiC) study found that limb pain is one of only four independent risk factors for meningococcal septicaemia. Therefore, leg pain should be recognised a 'red flag' symptom that has predictive value for meningococcal septicaemia by all paediatric nurses and clinicians.

Objectives The study aimed to explore the knowledge of members of the multidisciplinary team training and working in paediatrics in Wales regarding clinical features associated with meningococcal disease, particularly whether leg pain was understood to be a high-risk 'red flag' symptom.

Methods An online survey was completed by a convenience sample of paediatric nurses, doctors and final-year medical students. Demographic data was collected including job role (subspecialty where applicable), level of training and graduation date. Participants were asked about the estimated number of meningococcal septicaemia cases they encountered in their career.

Participants were asked to select whether a particular symptom or clinical sign was associated with meningococcal septicaemia or was alternatively non-specific. The signs and symptoms, listed in table 1 , were selected from the NICE clinical practice guideline [CG102] which lists specific signs of 